\title{
Deployment dynamics analysis of CALLISTO's approach and landing system
}

\author{
Anton Schneider ${ }^{1}\left[\right.$ ] Jean Desmariaux ${ }^{2} \cdot$ Josef Klevanski $^{3} \cdot$ Silvio Schröder $^{1} \cdot$ Lars Witte $^{1}$
}

Received: 23 September 2021 / Revised: 1 December 2021 / Accepted: 8 December 2021 / Published online: 18 December 2021

(c) The Author(s) 2021

\begin{abstract}
Prior to landing of reusable space transportation systems, the vehicle's landing legs needs to be fully deployed to enable a safe landing and further re-use of the space vehicle. During that phase the deployment system has to overcome harsh and challenging environmental conditions. In this study, a numerical simulator is developed in order to investigate these influences on the landing leg deployment dynamics. By means of an extensive aerodynamic database and a broad approach flight domain, the influence of aerodynamics, exhaust plume, and vehicle's attitude on the deployment dynamics is analyzed. This study shows on the example of the first stage demonstrator CALLISTO (Cooperative Action Leading to Launcher Innovation in Stage Toss back Operations), that thrust level, vehicle attitude, and the deployment system parameters affect the deployment performance.
\end{abstract}

Keywords Re-usability $\cdot$ Deployment $\cdot$ Simulation $\cdot$ Numerical analysis $\cdot$ CALLISTO $\cdot$ VTVL

\section{Introduction}

Driven by the need of cost reduction and new arising business concepts such as mega satellite constellations, conventional launcher business undergoes an major upheaval for the last decade. To serve this needs re-usability of launchers, which technological feasibility has already been successfully demonstrated by the US company SpaceX, seems to be promising for JAXA, CNES, and DLR. Hence, these space

Anton Schneider

anton.schneider@dlr.de

Jean Desmariaux

jean.desmariaux@cnes.fr

Josef Klevanski

josef.klevanski@dlr.de

Silvio Schröder

silvio.schroeder@dlr.de

Lars Witte

lars.witte@dlr.de

1 Present Address: Institute of Space Systems, German Aerospace Center (DLR), Bremen, Germany

2 Launcher Directorate, Centre National d'Etudes Spatiales (CNES), Paris, France

3 Institute of Aerodynamics and Flow Technology, German Aerospace Center (DLR), Cologne, Germany agencies jointly develop a reusable first stage demonstrator in the scope of a project, which is called CALLISTO (Cooperative Action Leading to Launcher Innovation in Stage Toss back Operations) [1]. CALLISTO is a reusable first stage demonstrator with a height of $13 \mathrm{~m}$ and a diameter of 1.1 $\mathrm{m}$. One key technology of CALLISTO is the Approach and Landing System (ALS), which is developed, designed and qualified by DLR. The knowledge thus obtained is subsequently transferred into the project called THEMIS [2].

CALLISTO as well as operational launcher and other reusable vertical take-off and vertical landing (VTVL) launcher under development cannot get along without a landing system, that is, due to efficiency reasons, stowed during ascent and descent phase, and gets deployed under atmospheric conditions shortly before touchdown. This approach phase is critical for the re-usability mission objectives, not only because an unsuccessfully deployed landing system will cause a vehicle loss, but also because an unfavorable deployment timing can have an influence on the vehicle's dynamics.

The present paper describes the development of the deployment dynamics simulator and aims to describe the impact of system and product design parameters on deployment performance of CALLISTO's approach and landing system. Furthermore, the following subsections show the 
process of creating aerodynamic database and the Approach Flight Domain from a system perspective.

\subsection{CALLISTO's approach and landing system}

The Approach and Landing System (ALS) is essential for a successful landing. Hence, the ALS needs to provide the capability to adsorb vehicle's kinetic energy at landing and limit the landing loads to the adjacent structures, such as CALLISTO's Aftbay. Another main function is to keep the landing vehicle in dynamic stable conditions during the entire landing phase and after touchdown for the postlanding and recovery phase $[1,3]$. Before the ALS is able to fulfill the landing related functions mentioned, it is necessary to deploy the stowed landing legs and hold them in deployed position, as shown in Fig. 1. In order to provide these functions, the ALS consists of a Primary Strut, Secondary Strut [4], and the deployment subsystem, that compromises a high pressure vessel, a deployment controller, a solenoid valve, pneumatic tubing, and several hold-down and release mechanisms (HDRM) and is located inside of CALLISTO's aft-bay. While the secondary strut assembly consists of passive parts, such as thermal protection system, foot pad, and CFRP tubes, the primary strut assembly consists of a telescopic structure that is able to extract. Two integrated latching mechanisms provide the capability to lock the telescopic segments in extended position, in order to prevent the telescopic structure of retraction. Energy absorbing elements are located in the primary strut assembly, which gets contracted at touchdown.

\subsection{Landing leg deployment system}

The deployment chain starts with opening the solenoid valve to the GHe-System to pressurize the ALS pneumatic subsystem. Opening of the Hold Down and Release Mechanisms (HDRM) allows the leg kinematics to unfold. The required actuation torque around the respective secondary strut hinge is initially provided by a push-off spring which drives the leg assemblies into a position where the pneumatic drive becomes effective. The landing gear controller is the device which orchestrates this event by turning the deployment signal from the On-Board Computer (OBC) into the electrical power distributed to the actuators. The deployment chain ends with stopping of the deployment motion by the mechanical end stops and the latching of the telescoping segments. The contributing equipment is described in the following paragraphs:

\subsubsection{Landing gear controller}

The Landing Gear Controller (LGC) is an electrical interface box which receives a deployment command (digital logic signal) from the on-board computer. This signal is used to open the solenoid valve letting pressure from the $\mathrm{GHe}$ pressure vessel building up in the feed lines. After a due delay time, the signal is acting on solid state relays to close the electrical circuit to the HDRMs. The HDRMs open their individual circuit when they release their mechanical pre-load.

\subsubsection{Hold Down Release Mechanisms}

The Hold Down and Release Mechanisms are commercial off-the-shelf components used to restrain the folded leg assembly against the aft-bay structure. Each leg assembly is locked by HDRMs against the aft-bay structure in stowed configuration, as shown in Fig. 1.

\subsubsection{Push-off mechanism}

When the legs are stowed the pneumatic pressure acts purely vertical and has no lever to force the landing leg sideways, it is kinematically blocked. Therefore a Push-Off mechanism
Fig. 1 Stowed (left) and deployed (right) configuration of ALS, the green arrow indicates the deployment process

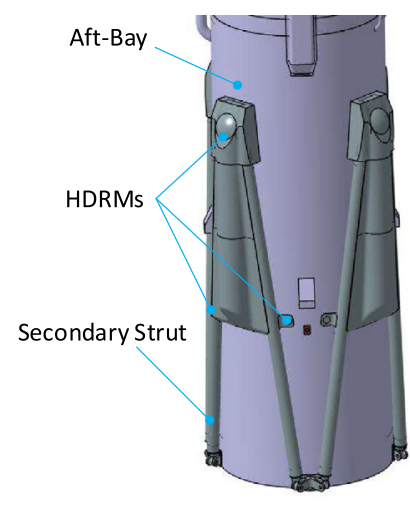


is necessary to overcome the first phase of unfolding the legs. The Push-Off mechanism consists of a set of torsion springs located at the end of the footpad. Those springs are connected to a lever that presses itself against vehicle's central core. After releasing the HDRMs this lever pushes the secondary strut away until it loses contact to the rocket. At this point the pneumatic force is strong enough to unfold the rest of the leg.

\subsubsection{Pneumatic equipment}

Unfolding the landing legs from a stowed to a deployed state is done by applying pneumatic pressure to the inner volume of the telescopic primary struts. The ALS pneumatic subsystem will use the gaseous Helium from the high pressure vessel. After the solenoid valve opens, the gas flows from the gas reservoir, through the solenoid valve into the landing legs, where it generates an over pressure. A pressure relief valve prevents the primary strut from overpressure. The solenoid valve is activated by electrical power coming from the Landing Gear Controller. After latching, the passivation of the deployment system is conducted passively through design features allowing the pressurized gas to escape from the primary strut inner volume.

\subsection{Approach flight domain}

CALLISTO Vehicle is foreseen to flight a large variety of flight profiles along which ALS deployment will occur, from high velocity to low velocity. As detailed mission definition was not available at the time of ALS deployment system design start, a systematic approach was developed to map the possible domain inside which ALS deployment might occur along flights, which is called "flight domain" in the following.

In order to do so, an analytical description of flight approach trajectories was developed that enable the construction of parametric synthetic trajectories, in terms of time, altitude, velocity and acceleration, and then derive flight parameters of importance for ALS performance study such as dynamic pressure and angle of attack.

The approach and landing trajectory was decomposed into two phases:

- Approach phase, during which vehicle cancels errors accumulated along flight. As a result, the flight profile features high dispersions.

- Landing phase, during which Vehicle prepares for touchdown. This phase features, by nature, much lower dispersions.

ALS deployment occurs during approach phase. This sequence transposes into a two steps flight profile which is illustrated in Fig. 2. The resulting approach flight domain consists of an array of nominal trajectories. The beginning of those trajectories is defined as the approach gate where propulsion system initiates deceleration. Convergence point is designated as "Approach Gate", which defines the handover from approach to landing phase. At this point, it is assumed that all major disturbances propagated until approach gate and generated during approach phase (e.g. ALS deployment) have been compensated, and that vehicle is prepared for final landing phase (last portion of the trajectory).

On Fig. 2, one can observe that approach phase flight domain is actually quite wide. This is linked to the fact that the deployment environment to be used for ALS deployment system design shall encompass flight conditions ranging from high energy (high velocity) to almost hovering conditions with an approach velocity lower than $1 \mathrm{~m} / \mathrm{s}$. On top of this, dispersions linked to a given flight profiles shall be added.

From this flight domain, and iterative design of the ALS deployment sequence was performed jointly between ALS and Vehicle System team so as to find right balance between the different constraints, through the assessment of various ALS deployment criteria along the provided trajectories.

\subsubsection{Deployment initiation}

The time point of the initiation of the deployment is defined by means of deployment conditions. Both cases, a deployment which is initiated too early or too late, results in challenging deployment conditions for the guidance, navigation and control (GNC) system, the vehicle structure as well as for the pneumatic deployment subsystem. For this study, the deployment conditions have been set to the

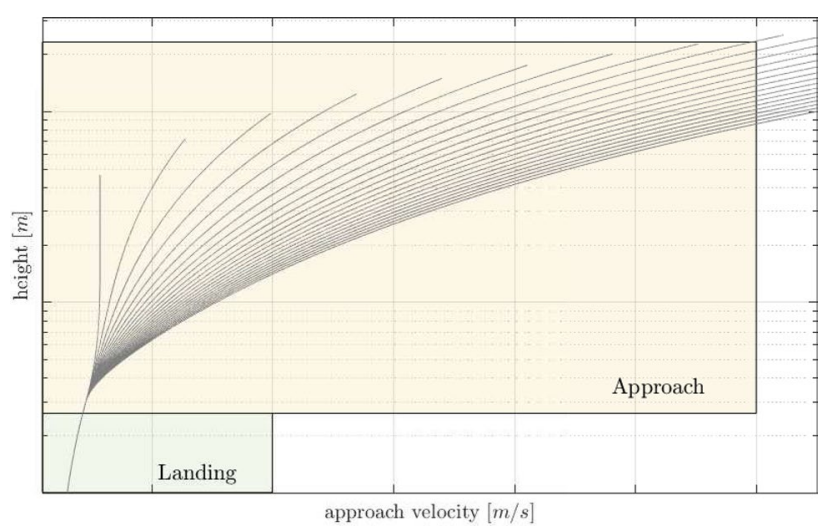

Fig. 2 Schematic definition of approach flight domain: Approach flight trajectories ranges from hovering like flight conditions with thrust to weight ratio of nearly 0 with velocities $<<1 \mathrm{~m} / \mathrm{s}$ (left hand side), to high energy flight conditions with maximum non-gravitational deceleration (right hand side) 
outer edges of the approach flight domain, which results in the following deployment condition:

$\delta_{\mathrm{dpl}}\left(t_{\mathrm{td}}, V_{\mathrm{clt}}\right)= \begin{cases}1 & \text { if } t_{\mathrm{td}} \leq-8 \mathrm{~s} \wedge V_{\mathrm{clt}} \geq-60 \mathrm{~m} / \mathrm{s} \\ 0 & \text { if } t_{\mathrm{td}} \geq-8 \mathrm{~s} \vee V_{\mathrm{clt}} \leq-60 \mathrm{~m} / \mathrm{s}\end{cases}$

Deployment conditions are of crucial importance, since the time point of deployment initiation directly affects the magnitude and duration of disturbance torques acting on the vehicle. In addition, deployment conditions have an impact on the internal shock loads, as, for example, a late deployment at lower aerodynamic forces lead consequently to higher deployment velocity, and thus to higher structural shock, that the landing leg structure needs to withstand.

\subsubsection{Deployment time line}

The deployment phase is initiated by the on-board computer and processed by the ALS Controller into the following event time line:

1. Opening of solenoid valve

2. Release of HDRM \#2 and \#3

3. time delay

4. Release of HDRM \#1

5. Kinematic deployment of landing legs

The deployment phase is finished when all legs are fully deployed. A detailed event time line is illustrated in Fig. 3.

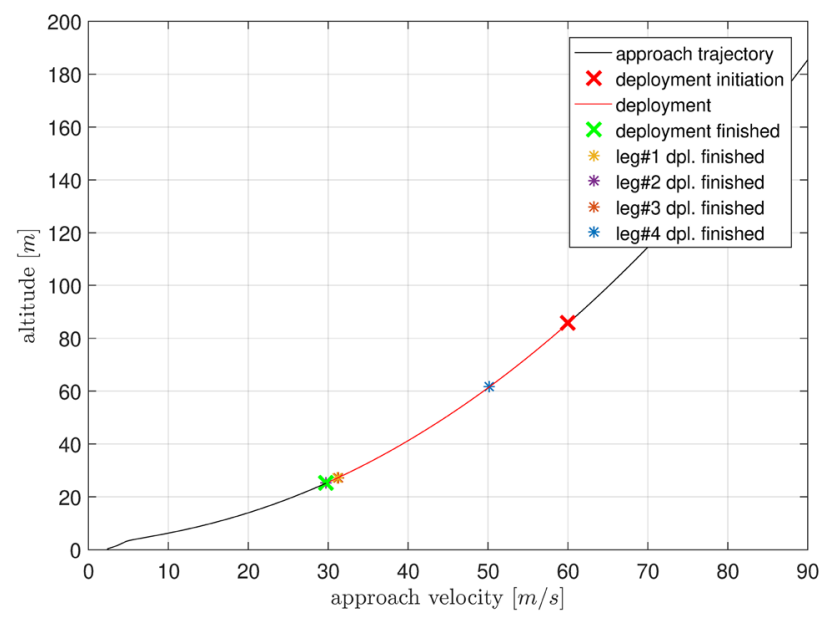

Fig. 3 Event time line graph of an exemplary trajectory of the approach flight domain

\section{Numerical model of the ALS deployment system}

The simulator is subdivided into sub-models, that corresponds with ALS components described in previous section. Additionally, an extensive aerodynamic database (AEDB), that consists of local aerodynamic coefficients and covers external aerodynamic influences, is implemented in the deployment simulator. Inertial loads, due to vehicle's acceleration during the approach phase, are provided to the deployment simulator by the approach flight domain database.

The deployment logic sub-model receives the vehicle's state vector $\underline{X}$, which contains attitude, altitude and velocity of the vehicle, from the Approach Flight Domain Database. When deployment conditions are met, the deployment is initiated and the deployment state $\delta_{\mathrm{dpl}}$ is transmitted to the kinematics and pneumatics model block, where the landing leg angular relations are calculated and thermodynamic state of the working gas is determined, respectively. Consequently, the actuation torque $\underline{M}_{\mathrm{ps}}^{h}$ is feed forwarded to the kinetics block. Besides the deployment state $\delta_{\mathrm{dpl}}$, the pneumatic model receives information about the landing leg expansion volumes $\underline{V}$ and the landing angle $\epsilon$, which depend on the opening angle $\lambda$ and is processed by the landing leg's kinematics sub-model. The aerodynamic hinge torques $\underline{M}_{\text {aero }}^{h}$ depend on both the vehicle's state vector $\underline{X}$ and the landing leg opening angle $\underline{\lambda}$ and are determined by the AEDB and passed to the kinetics sub-model. Initial push-off torque $\underline{M}_{\mathrm{p} / \mathrm{o}}^{h}$ is provided by the push-off mechanism sub-model, which receives the opening angle $\lambda$ as an input variable. Vehicles accelerations that affect the deployment dynamics $\underline{M}_{\text {acc }}^{h}$ are defined by the approach and flight domain sub-model and passed to the kinetics sub-model.

In this sub-model, equation of motions of each landing leg are modeled and processed. The main inputs are the torque components $\underline{M}^{h}$, which are provided by corresponding sub-models, as shown in Fig. 4. Functionality and governing equations of these sub-models are described in the following sections.

The landing leg assembly have been simplified to reduce the complexity of the model and, therefore, reduce calculation time. For this purpose, it is assumed that the Landing Leg assembly mass is concentrated in a point mass (Center of Gravity) with a constant moment of inertia, although the geometry is changing during deployment process. Due to the kinematic constraints of the secondary strut, the combined mass is only able to move around the hinge of the secondary strut, as shown in Fig. 5. The rotatory motion of a point mass can be described with the angular momentum equation: 
Fig. 4 Simplified deployment simulator block diagram (first layer)

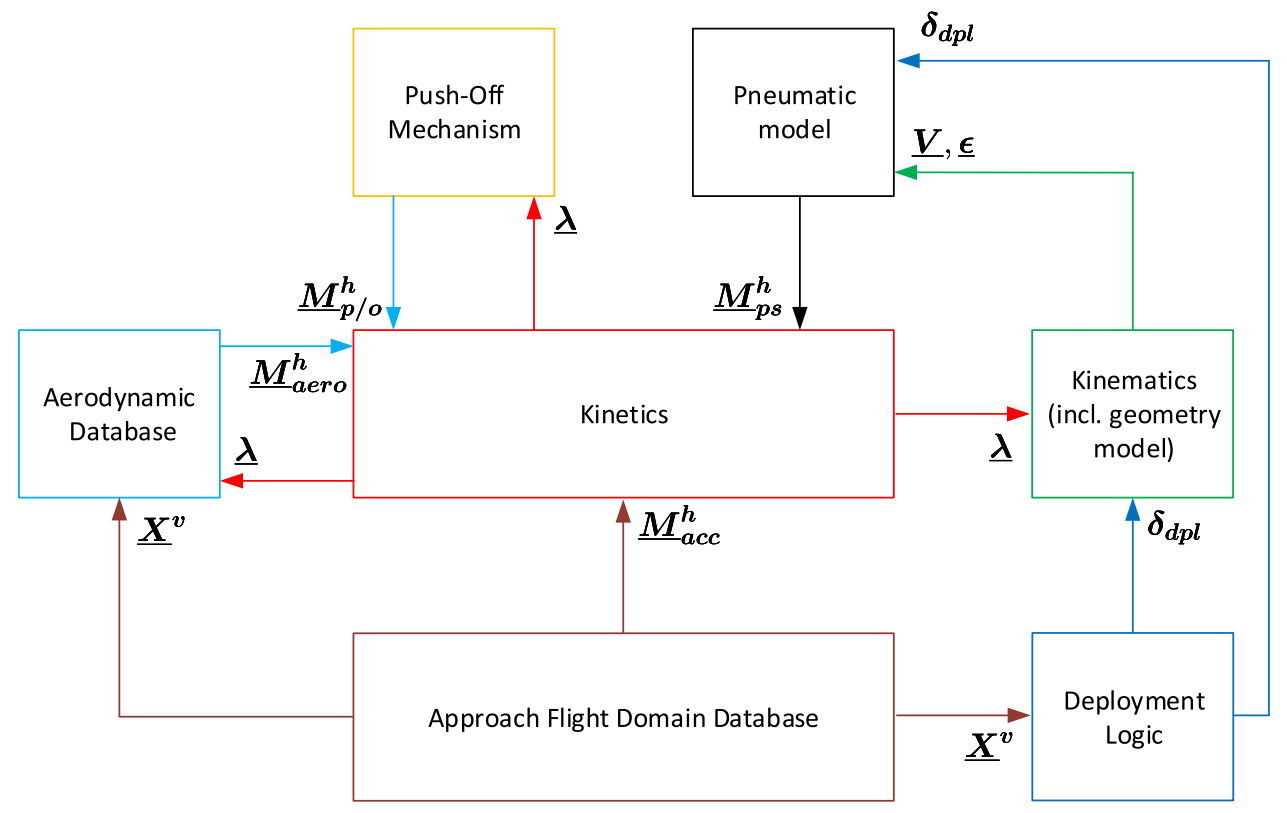

Fig. 5 Simplified view of the model (left) and orientation of forces and trajectory parameters (right)

$\underline{\dot{L}}^{h}=\left[\begin{array}{l}L_{1} \\ L_{2} \\ L_{3} \\ L_{4}\end{array}\right]=\sum_{k=1}^{n} \underline{M}_{k}^{h}=I_{z z}^{h} \cdot \underline{\lambda}^{h}$
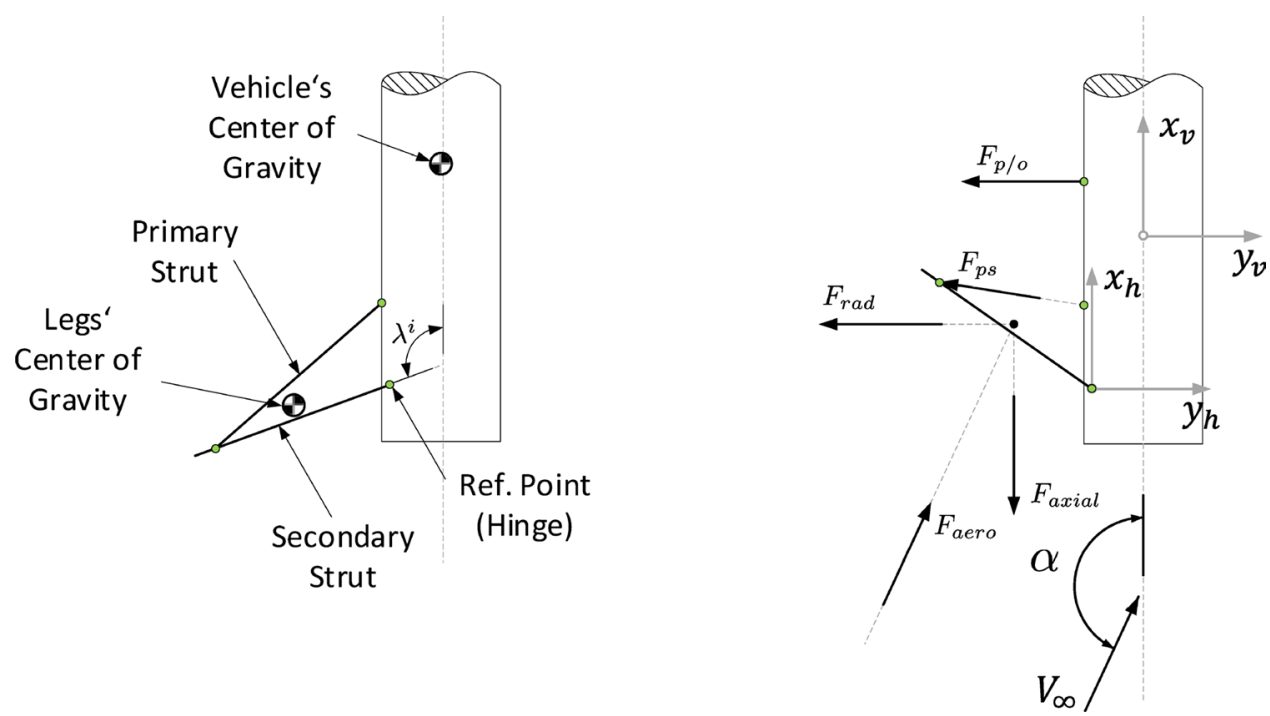

with $\sum_{k=1}^{n} \underline{M}_{k}^{h}$ the sum of all torque components acting on a single leg, $I_{z z}^{h}$ the moment of inertia, and $\underline{\ddot{\lambda}}^{h}$ the legs angular acceleration. The external and internal forces that are contributing to the deployment are mainly caused by the aerodynamics, the trajectory profile and the actuation of the primary strut. These forces generate a total torque

$\sum_{k=1}^{n} \underline{M}_{k}^{h}=\underline{M}_{\mathrm{aero}}^{h}+\underline{M}_{\mathrm{ps}}^{h}+\underline{M}_{\mathrm{p} / \mathrm{o}}^{h}+\underline{M}_{\mathrm{acc}}^{h}$ that consists of

- $M_{\text {aero }}^{h}$ the aerodynamic torque,

- $M_{\mathrm{ps}}^{h}$ the primary strut torque,

- $M_{\mathrm{p} / \mathrm{o}}^{h}$ the push-off mechanism torque, and

- $M_{\mathrm{acc}}^{h}$ the torque, due to vehicle's acceleration.

The location and orientation of forces, that generates the torques mentioned in the listing above, are illustrated in Fig. 5.

Push-off mechanism model Due to the mechanical design of the push-off mechanism, the provided hinge torque 
$\underline{M}_{\mathrm{p} / \mathrm{o}}= \begin{cases}\left(F_{\mathrm{p} / \mathrm{o}, \max }-\frac{F_{\mathrm{p} / \mathrm{o}, \max }}{\lambda_{\mathrm{p} / \mathrm{max}}} \underline{\lambda}\right) l_{s s} & \text { if } \underline{\lambda} \leq \lambda_{\mathrm{p} / \mathrm{o}, \text { max }} \\ 0 & \text { if } \underline{\lambda}>\lambda_{\mathrm{p} / \mathrm{o}, \text { max }}\end{cases}$

is assumed to be linearly dependent on $\underline{\lambda} \cdot \lambda_{\mathrm{p} / \mathrm{o} \text {,max }}$ defines the point, where the push-off mechanism loses contact, and, therefore, cannot generate any torque from this point on. Also, once reached $\lambda_{\mathrm{p} / \mathrm{o} \text {, max }}$ the opening angle $\lambda$ cannot fall below that value, due to design restrictions. The max. force at $\lambda=0^{\circ}$ is a key design parameter and defined as $F_{\mathrm{p} / \mathrm{o}, \max }$.

\subsection{Pneumatic power supply system}

The pneumatic circuit model consists of 6 control volumes, that are interlinked as shown in Fig. 6. Control volumes \#1 to \#4 represents the landing leg volumes, whereas the pressure vessel and ring line volume is named with \#5 and \#6. These control volumes are connected with lines, that routes the working gas from the pressure vessel to the landing leg's expansion volume and vice versa. While control volumes \#5 and \#6 are of constant volume, the landing leg volumes \#1 to \#4 change their size with the opening angle $\lambda$. According to the ideal gas law, the pressure inside of control volumes is defined as:

$p=\frac{m}{V(\lambda)} R T$

where $p$ is the pressure, $V$ is the control volume, $R$ is gas constant, e.g. for helium, $T$ is the gas temperature, and $i$ represents the node number. Based on this, the mass flow rate between interconnected control volumes $i$ and $j$ is defined as [5]

$\dot{m}^{i, j}=A \cdot \Psi^{i, j} \cdot \sqrt{2 \cdot p^{i} \cdot \rho^{i}}$

with the psi-flow function:

$$
\Psi^{i}= \begin{cases}\sqrt{\frac{\kappa}{\kappa-1}\left[\left(\frac{p^{j}}{p^{i}}\right)^{\frac{2}{\kappa}}-\left(\frac{p^{j}}{p^{i}}\right)^{\frac{\kappa+1}{\kappa}}\right]} & \text { if } \frac{p^{j}}{p^{i}}>p_{\text {crit }} \text { (subsonic flow) } \\ \left(\frac{2}{\kappa+1}\right)^{\frac{1}{\kappa-1}} \sqrt{\frac{\kappa}{\kappa+1}} & \text { if } \frac{p^{j}}{p^{i}} \leq p_{\text {crit }} \text { (chocked flow) }\end{cases}
$$

where $p^{j} / p^{i}$ is the pressure ratio of control volumes $i$ and $j$, $\kappa$ the heat capacity ratio, and $p_{\text {crit }}$ the critical pressure ratio, at which the flow reaches sonic velocity and chokes. In addition, the gas in each of the control volumes need to fulfill the mass conservation equation

$\int_{V} \rho \mathrm{d} V=\int_{t}\left(\dot{m}_{\mathrm{in}}-\dot{m}_{\mathrm{out}}\right) \mathrm{d} t$

and the energy conservation equation:

$\mathrm{d} U=\delta Q+\delta W$

After several transformation steps, the Eqs. 8 and 9 lead to the thermal model equation [6]:

$$
\begin{gathered}
\dot{m}^{i, j} \cdot T^{j}+m^{j} \cdot \dot{T}^{i, j}=\kappa T^{j} \cdot \dot{m}^{i, j}-\kappa T^{j} \cdot \dot{m}^{i, j} \\
-\frac{p^{j} \cdot \dot{V}^{j}}{C_{v}^{j}}-\frac{h^{j} \cdot a^{j} \cdot\left(T^{j}-T_{\mathrm{amb}}\right)}{C_{v}^{j}}
\end{gathered}
$$

This set of partial differential equations (PDE) allows to numerically calculate the gas state inside of each of the control volumes, which then is used to define the force of the primary strut acting on the secondary strut, as follows

$\underline{F}_{\mathrm{ps}}=\left(\underline{p}-p_{\mathrm{amb}}\right) \cdot \underline{A}_{\mathrm{ps}}(\underline{\lambda})$

with $p_{\text {amb }}$ the atmospheric pressure, $p_{i}$ the control volume pressure, and the $A_{i, \mathrm{ps}}^{i}$ projected area of the primary strut.
Fig. 6 Pneumatic circuit model of the ALS, blue arrows indicated the allowed flow directions

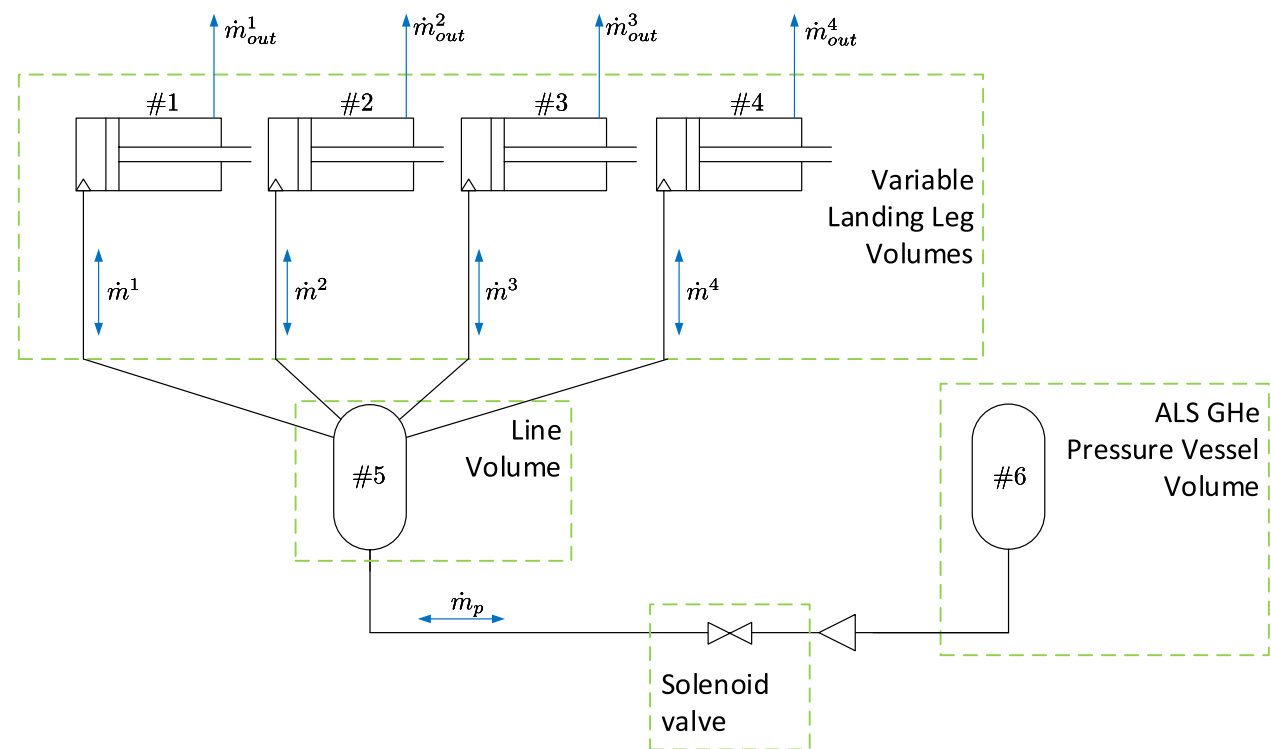


The projected area has two states and changes after the first latching occur, due to the design of the primary strut. Then $A_{\mathrm{ps}}^{i}$ is reduced by the size of the projected area of the second segment:

$A_{\mathrm{ps}}^{i}= \begin{cases}A_{s 2}+A_{s 3} & \text { if } \lambda^{i} \leq \lambda_{\operatorname{lm} 12} \\ A_{s 3} & \text { if } \lambda^{i} \geq \lambda_{\operatorname{lm} 12}\end{cases}$

Taking the kinematic into account, the hinge torque due to primary strut pressurization can be calculated as follows:

$\underline{M}_{\mathrm{ps}}(t, \underline{\lambda})=\underline{F}_{\mathrm{ps}}(t, \underline{\lambda}) \cdot \sin (\underline{\epsilon}) \cdot l_{s s}$

The mass flow rate $\underline{\dot{m}}_{\text {out }}$ is the sum of mass flow rate of the pressure relief valve $\underline{\underline{m}}_{\text {relief }}$ and of the safety venting feature $\dot{\underline{m}}_{\text {vent }}$.

$\underline{\dot{m}}_{\mathrm{out}}=\underline{\dot{m}}_{\mathrm{relief}}+\underline{\dot{m}}_{\mathrm{vent}}$

To prevent the assembly from unintended pressure peaks a pressure relief valve is located in each landing leg assembly. In case the leg pressure exceeds the pressure relief threshold, then the valve opens automatically, so that the leg pressure can decrease.

$\dot{m}_{\text {relief }}= \begin{cases}0 & \text { if } \underline{p} \leq p_{\max } \\ \text { Equation } 6 & \text { if } \underline{p} \geq p_{\max }\end{cases}$

A passive safety venting design feature has been foreseen, to allow the system to depressurize after a successful deployment. The way the safety venting feature have been designed ensures that the pressure decay curve is sufficient flat to prevent the system from unintended depressurization during flight.

$\underline{\dot{m}}_{\mathrm{vent}}= \begin{cases}0 & \text { if } \underline{\lambda} \leq \lambda_{\max } \\ \text { Equation } 6 & \text { if } \underline{\lambda} \geq \lambda_{\max }\end{cases}$

\subsection{Aerodynamic database}

An aerodynamic database (AEDB) has been created, to assess the effect of aerodynamics on the entire vehicle and especially on unfolding dynamics of each landing leg during CALLISTO's approach phase. The complex aerodynamics of CALLISTO vehicle have been discussed in previous study [7], though the main goal of this study is to cover the influences of thrust level, angle of attack AoA, angle of roll AoR, leg's opening angle $\lambda$, and Mach number Ma on the aerodynamic forces acting on individual landing leg. The interaction of these influences at one point along a approach trajectory is illustrated in Fig. 7.

Since analytical engineering methods are not able to consider CALLISTO's geometry and external influences, such as engine plume, multiple three-dimensional CFD simulations have been performed. All calculations are based on Euler-equations and are performed with DLR in-house developed TAU solver [8]. Calculated parameter ranges are the following: $\mathrm{Ma}=0.266$ to $\mathrm{Ma}=0.5, \mathrm{AoA}=160^{\circ}$ to $\mathrm{AoA}$ $=200^{\circ}, \mathrm{AoR}=-45^{\circ}$ to $\mathrm{AoA}=90^{\circ}$, opening angle $\lambda=20^{\circ}$ to $\lambda=115^{\circ}$, and thrust range from $0 \%$ to $100 \%$. The engine plume was modeled with a two-gas mixture, without considering chemical reactions. The resulting aerodynamic data are processed to a multi-dimensional look up table, that acts as input for the deployment simulator and mainly contains the aerodynamic hinge torque- and force coefficients for each landing leg.

Aerodynamic coefficients for flight parameter between the grid points of the AEDB are interpolated linearly, whereas for flight parameter combinations outside of the calculated parameter range the nearest grid point is taken (no extrapolation). The aerodynamic force- and torque-coefficients of a single leg with respect to the hinge-frame depends on the Mach number $M a$, the angle of attack $\alpha$, the angle of roll $\phi$, the opening angle $\lambda$, and the thrust level Thr:
Fig. 7 Ambient flow density (left) and pressure distribution (right) around the ALS landing legs for $\mathrm{AoR}=0^{\circ}, \mathrm{AoA}=155^{\circ}$, $\lambda=90^{\circ}$ and $\mathrm{Ma}=0.266$

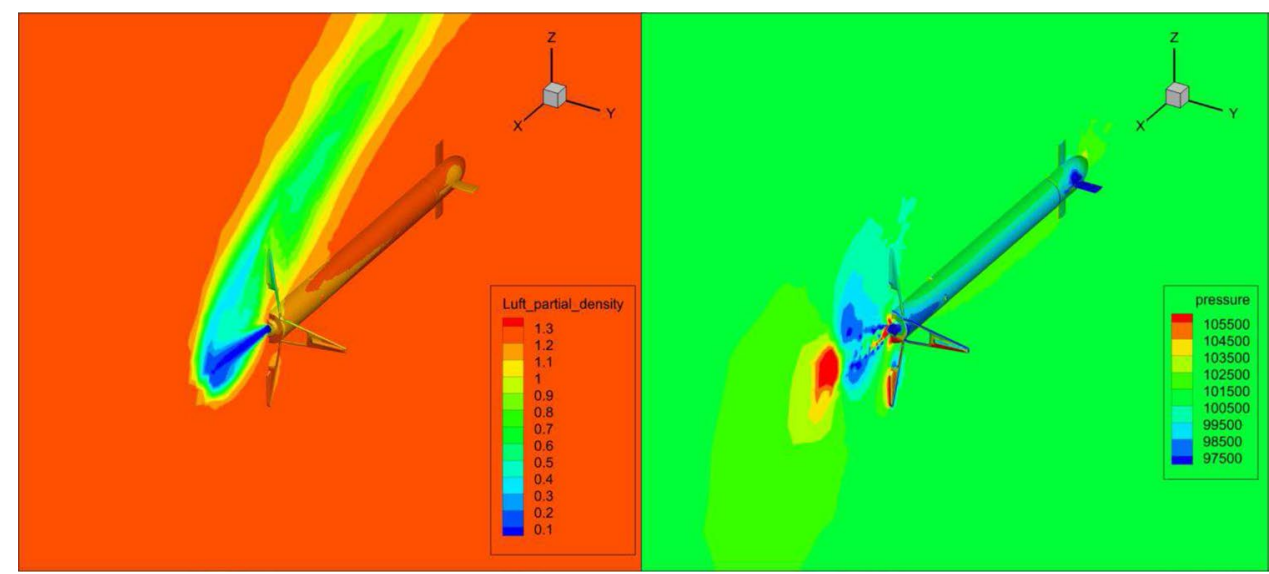


$\underline{C}_{\mathrm{M}}^{h}=f(\mathrm{Ma}, \alpha, \phi, \underline{\lambda}, \mathrm{Thr})$

The hinge torque coefficient $\underline{C}_{\mathrm{M}}^{h}$ with its multi-dimensional dependencies is displayed in Fig. 8. Further, the aerodynamic torque with respect to hinge coordinate frame is defined as

$\underline{M}_{\text {aero }}^{h}=\frac{1}{2} \rho_{\infty} V_{\infty}^{2} A_{\text {ref }} \underline{C}_{\mathrm{M}}^{h} l_{\text {ref }}$

where $V_{\infty}$ is the approach velocity, $\rho_{\infty}$ is the atmospheric density, and $A_{\text {ref }}$ reference area.

\section{Landing leg deployment}

\subsection{Single leg deployment dynamics analysis}

Shortly after deployment initiation, the working gas begins to flow first into the tubing and then into the landing leg's volumes, due to the pressure difference between the pressure vessel and the pneumatic system components. The increase in gas mass in leg's volumes leads to an increase in gas pressure and, therefore, in a force that acts on the hinge between primary and secondary strut. Hence, the landing leg unfolding begins, the opening angle $\lambda$ increases. Due to kinematic conditions the primary strut extends and the inner expansion volume increases. According to the ideal gas law, shown in Eq. 19, the ratio between the mass flow into the leg's volume and the change rate of the leg's volume is proportional to the leg's pressure.

$\underline{p}=\frac{m}{\underline{V}} R_{\mathrm{gas}} \underline{T}$

As described in Sect. 2.1, the pressure $p$ acts on the secondary strut and, hence, contributes to the unfolding of the landing leg. In order to overcome the kinematic blocking of the primary strut, a push-off mechanism is foreseen to provide the initial torque, until the angle $\epsilon$ between primary and secondary strut reaches a value, that enables the primary strut force to overcome the external load. These two torques are product design parameter, which mainly contributes to the deployment of the landing legs.

Aerodynamic forces generate a counter torque, that acts against the deployment direction of the landing leg. When the landing leg is located on the lee site, then aerodynamic forces support the deployment for a broad range of $\lambda$.

The vehicle's axial acceleration supports the opening of all landing legs during the approach phase, due to its parallel orientation with the $x_{h}$ axis. Whether the lateral acceleration contributes to the opening of landing legs, depends on the angle $\psi$, which is defines the mounting position of the landing legs.
Fig. 8 Hinge torque coefficients of the ALS landing legs for AoR $=0^{\circ}$ (left), AoR $=45^{\circ}$ (right) and a thrust level of $40 \%$

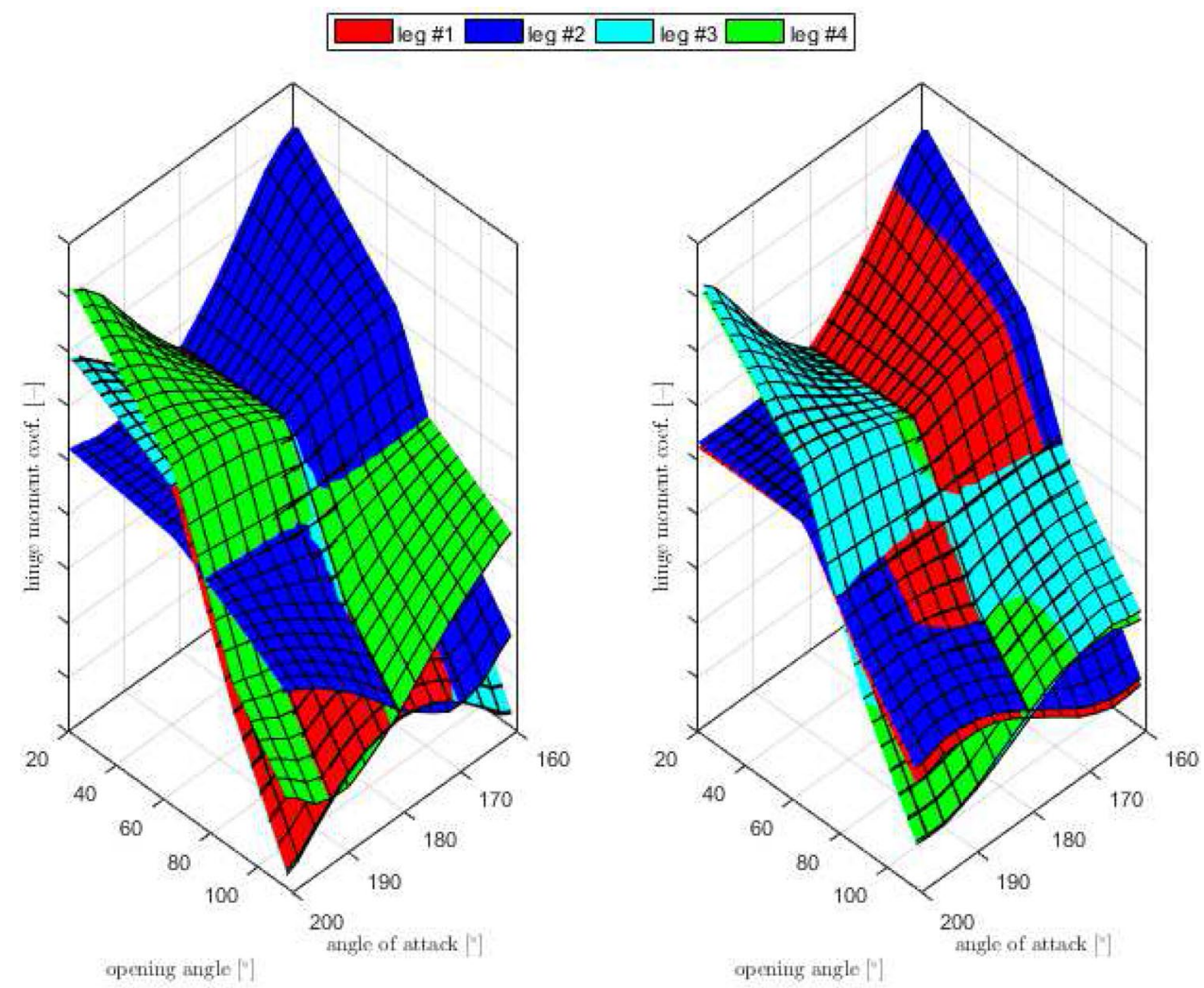


$\underline{M}_{\text {acc }}^{h}=m_{l}\left(a_{\text {lateral }} \cdot \sin (\underline{\psi})+a_{\text {axial }}\right)$

Hinge torque contributors as a function of the opening angle $\lambda$ for an exemplary deployment case are shown in Fig. 9.

After releasing the HDRMs, a degree of freedom is added into the system, which allows the landing leg to rotate around the opening axis $z^{h}$. The lower part of Fig. 10 shows exemplary the opening angle $\lambda$ and its time derivative $\dot{\lambda}$ as a function of time. Here, the deployment is initiated at approximately $6 s$ and finishes at approximately $3.8 s$. The rotational velocity has its peak in the first part of the deployment. Up to this point, the sum of push-off torque and primary strut torque is greater than the demand torque, which results in high acceleration and, hence, large rotational velocities. This is because the effective angle of attack of the landing leg is equal to the opening angle, which is small at the beginning of the deployment phase. Hence, the area that is subjected to the flow is small and consequently leads to small aerodynamic forces.

Passing that point, the aerodynamic torque increases constantly and decelerates the leg. Simultaneously after the rotational velocity peak, the pressure ratio $p^{i} / p^{5}$ for $i \leq 4$ decreases to its minimum, because the ratio of continuous increase of expansion volume of the primary strut and the mass flow rate is, in this case, not sufficient to keep the pressure on a constant level. Consequently, inner forces and outer loads try to attain equilibrium.

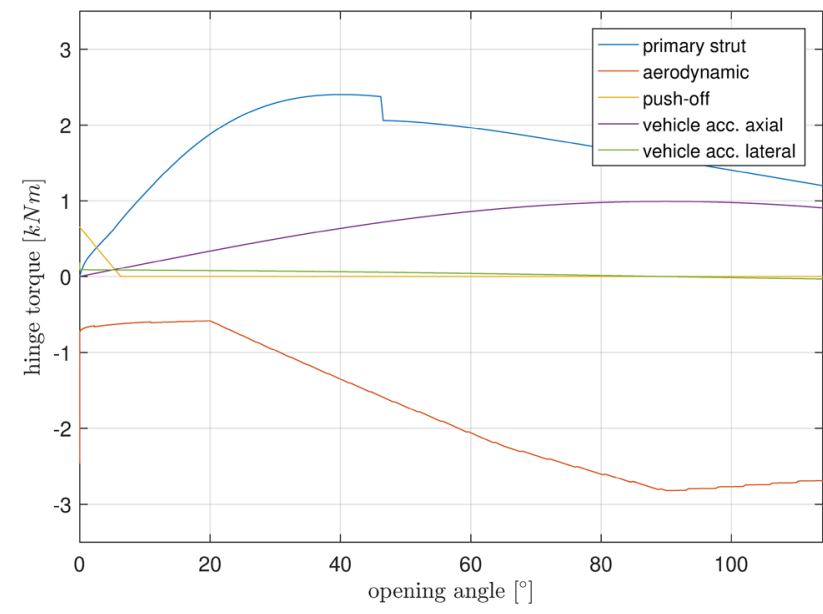

Fig. 9 Hinge torque components as a function of opening angle $\lambda$. Please note: According to the coordinate system definition, torques with positive sign support the opening of a landing leg, whereas negative signs contribute to the counter hinge torques, which act against the deployment direction of the landing leg
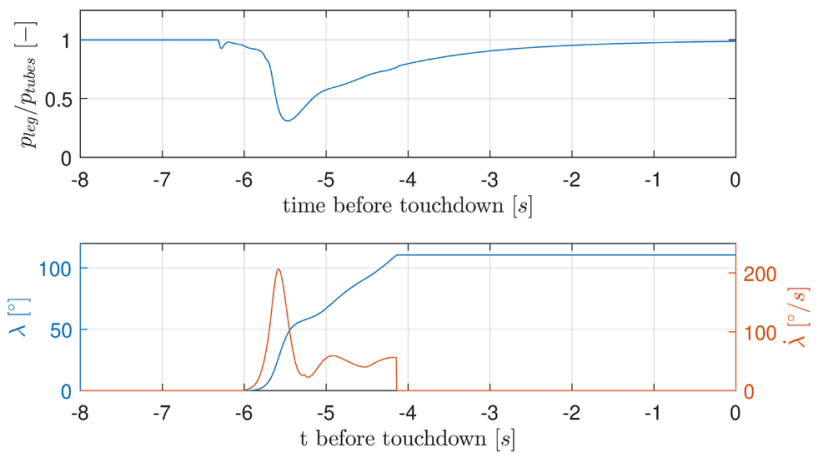

Fig. 10 Pressure ratio of landing leg pressure and line pressure (top) and corresponding opening angle and velocity (bottom)

\subsection{Four leg deployment dynamics analysis}

The ALS consists, among others, of four landing leg assemblies, that are interlinked via pneumatic lines. This system architectural feature allows the working gas to flow between the control volumes in both directions, and, thus balance out pressure differences of aforementioned control volumes. The gas, for example, trapped in one leg can flow back to the ring line, in case this landing leg retracts and the leg's pressure exceeds ring line pressure. Consequently, the landing leg deployment dynamics are mutually dependent.

This dependency is reflected in Fig. 11 (bottom part), where the pressure ratio of landing leg to line pressure is shown. Here, the pressure drops at the deployment initiation, because the pressure in the line volume increases faster than the pressure in the larger legs volumes. Then, after a short delay the HDRMs release the legs, and, in this example, landing leg number \#1 and \#4 begin their movement, because of their leeward orientation. In parallel, leg number
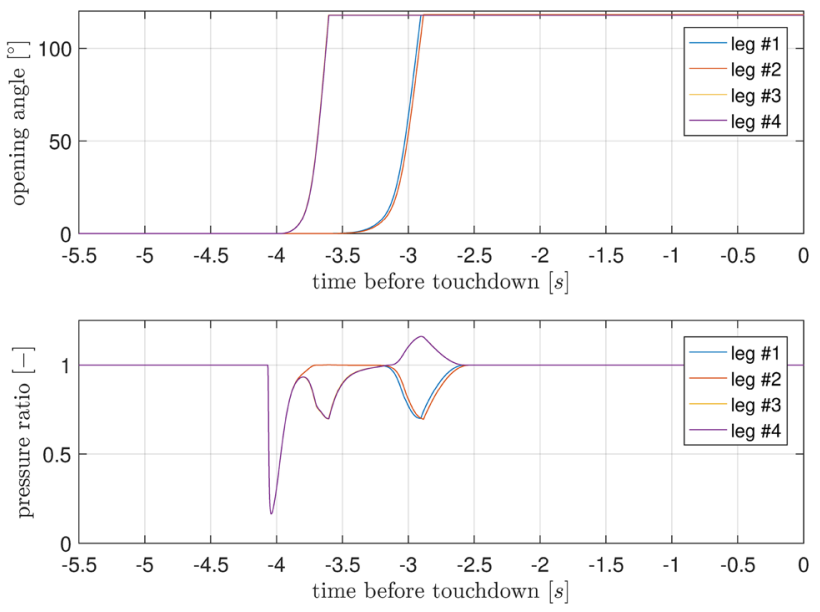

Fig. 11 Pressure ratio of landing legs pressure and line pressure (top) and corresponding opening angle (bottom) of all legs 
\#2 and \#3, which are facing windward, do not move, which is indicated by a pressure ratio of 1 .

\subsubsection{Deployment performance}

Within the development of a landing leg deployment system and to provide comparability of different parameter sets it is useful to define success criteria, that enables comparability between two different system parameter sets. The following two success criteria have been defined within the scope of this study:

- Total deployment duration shall not exceed $t_{\mathrm{dpl}}$, max

- Deployment shall not finish later than $t_{\text {latest }}$

Other criteria can be applied from the structural and GNC perspective, in order to analyze the disturbance torques and the kinetic energy at latching. With this set of criteria and, for the sake of completeness, with the deployment initiation parameters it is possible to analyze the deployment performance in the scope of the approach flight domain. Fig. 12 shows the approach flight trajectories with deployment initiation time points, as well as the time point of deployment finish. A deployment is finished, when all legs are fully deployed, hence the deployment duration is defined as:

$t_{\mathrm{dur}}=t_{\mathrm{ini}}-\operatorname{latest}\left(t_{\mathrm{dpl}}\right)$

with the time point of deployment initiation $t_{\text {ini }}$, and the time point of latest leg unfolding latest $\left(t_{-\mathrm{dpl}}\right)$. As a result, a window, where the deployment process can take place, is defined and allows to compare the deployment performance of two different system parameter and product parameter sets.

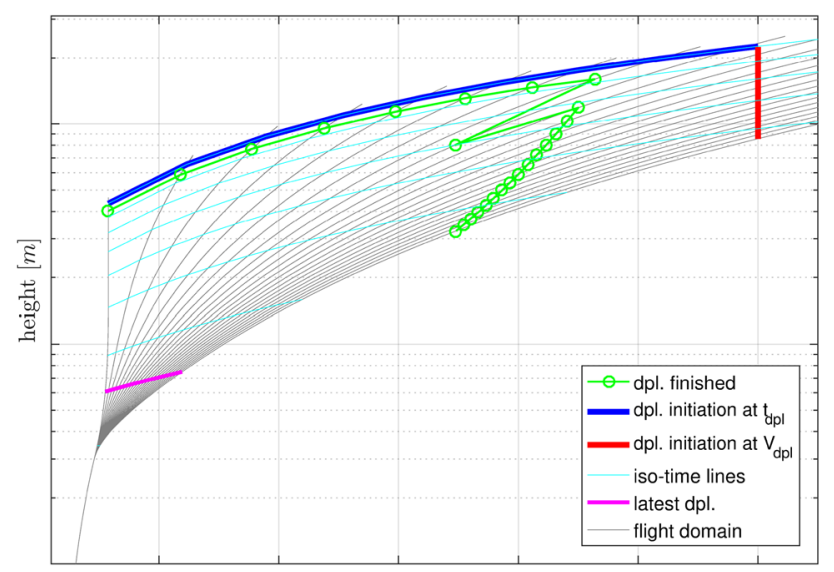

approach velocity $[\mathrm{m} / \mathrm{s}]$

Fig. 12 Exemplary deployment performance graph

\subsection{Disturbance torques and forces}

During deployment each landing leg generates forces and torques, and hence disturbance torques, in case the force and torque components of two opposed landing legs do not cancel each other out. These disturbances need-in general-to be avoided or minimized, to reduce the stress on the GNCsystem, which need to compensate thus generated torque $\mathbf{T}^{v}$ and force $\mathbf{F}^{v}$ vectors:

$\mathbf{T}^{\nu}=\sum_{i=1}^{n=4} \mathbf{M}^{i, v}$

$\mathbf{F}^{v}=\sum_{i=1}^{n=4} \mathbf{F}^{i, v}$

One reason for the occurrence of disturbance torques and forces is the asynchronous deployment, which results from a force and torque imbalance of the external load (demand) and the internal supply forces, which is in case of CALLISTO for each landing leg equal. The main reason for differences between loads acting on landing legs is the asymmetric flow condition around the re-entry vehicle. Angle of Attack trajectory profiles, that are not equal to $180^{\circ}$, creates a slipstream and, in addition, redirects the engine plume toward a single landing leg. Both effects create a force imbalance of the landing legs, and consequently a asynchronous deployment.

The reduction of external force variances is practically not possible, as even during an approach phase with infinitesimal small AoA and lateral acceleration the thrust vector needs to be deflected for vehicle control reasons. Consequently, thrust vector gimballing redirects the plume and contributes to external force imbalance. In contrast, however, internal forces can be reduced by over-sizing the pneumatic power, so that other contributors, such as friction forces, become negligible compared to the pressure force.

Aerodynamic forces in axial vehicle direction are byproduct of the deployment and, in contrast to lateral forces, beneficial, because they contribute to the deceleration of the vehicle, while approaching to the landing site. A maximization of the flight duration with fully deployed legs could be advantageous for the propellent budget, but needs to be confirm with other system requirements.

Depending on the flight trajectory and deployment conditions definition during approach phase, the force and torque vector composition changes. In general, an approach with symmetric flight orientation $\left(\mathrm{AoA} \approx 0^{\circ}\right.$ and $\mathrm{AoR} \approx 0^{\circ}$ ) generates lower disturbance torques and forces. Fig. 13 shows an exemplary graph of disturbance torques and forces, based on CALLISTO's approach phase with an AoR $=45^{\circ}$ profile. In this flight orientation two legs face the undisturbed 
Fig. 13 Disturbance Torques (upper part) and forces (lower aprt) for AoR $=0^{\circ}$ (left) and AoR $=45^{\circ}$ (right). Please note, $\mathrm{x}$-component of disturbance torque vector is negligible small for the AoR mentioned
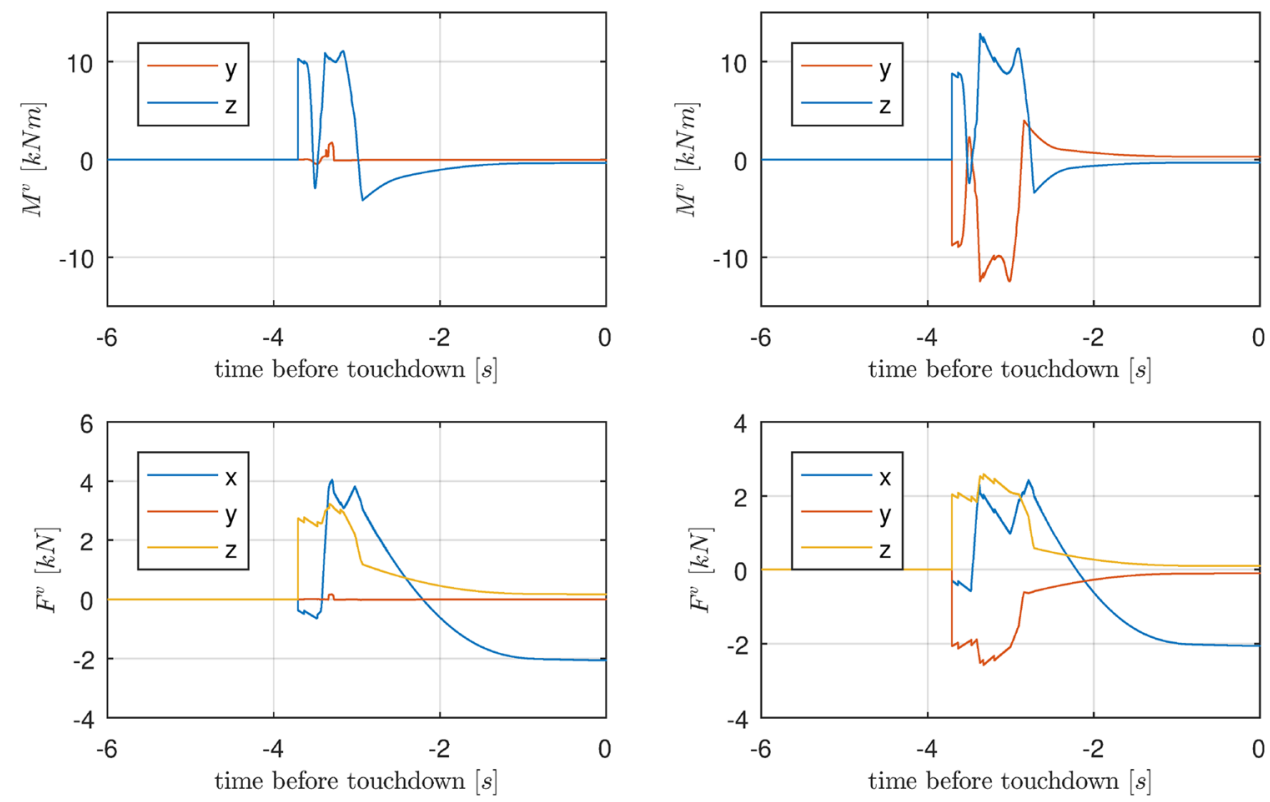

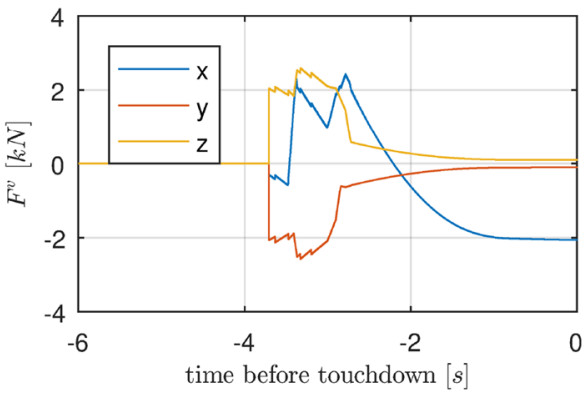

flow and the opposing legs are located in the slipstream of CALLISTO, which generates the highest torque and force imbalances. Change of sign of the axial component of the disturbance force vector $\mathbf{F}^{v}$ signifies the point, where the inertial forces $F_{\text {acc }}^{v}$ overcome the aerodynamic force $F_{\text {aero }}^{v}$.

While an AoR of $n \cdot 45^{\circ}$ mainly contribute to the $z$ and $y$ component of $\mathbf{T}^{v}$, any value in between takes influence on the $x$ component of the disturbance torque vector. To study the impact of the disturbance torques on the vehicle dynamics, the deployment simulator and the vehicle dynamics simulator needs to be coupled.

\subsection{Influence of the angle of roll on deployment performance}

The Angle of Roll (AoR) defines the rotational position of the launch vehicle with respect to the aerodynamic coordinate system. Consequently, the AoR affects the flow conditions around the re-entry vehicle, and, therefore, the plume direction, local aerodynamics and the orientation of the lateral acceleration vector. Since the AoR can take any possible value, this study focuses on two AoR that are representative for extreme plume orientations.

- AoR of $0^{\circ}$ : One leg is fully oriented windward and the opposite leg is oriented leeward. In addition, the engine plume core hits the leeward oriented leg.

- AoR of $45^{\circ}$ : Two landing legs are partially facing leeward and the opposing two landing legs are partially oriented windward. In this case the engine plume core passes between the leeward oriented legs, but non the less reduces the landing leg's aerodynamic coefficients.

The flow field of these two assessed flight orientation is illustrated in Fig. 14.
Fig. 14 Flow field comparison of AoR $0^{\circ}$ (left) and AoR 45 (right) flight configuration. The ISO-density surface illustrates the engine plume

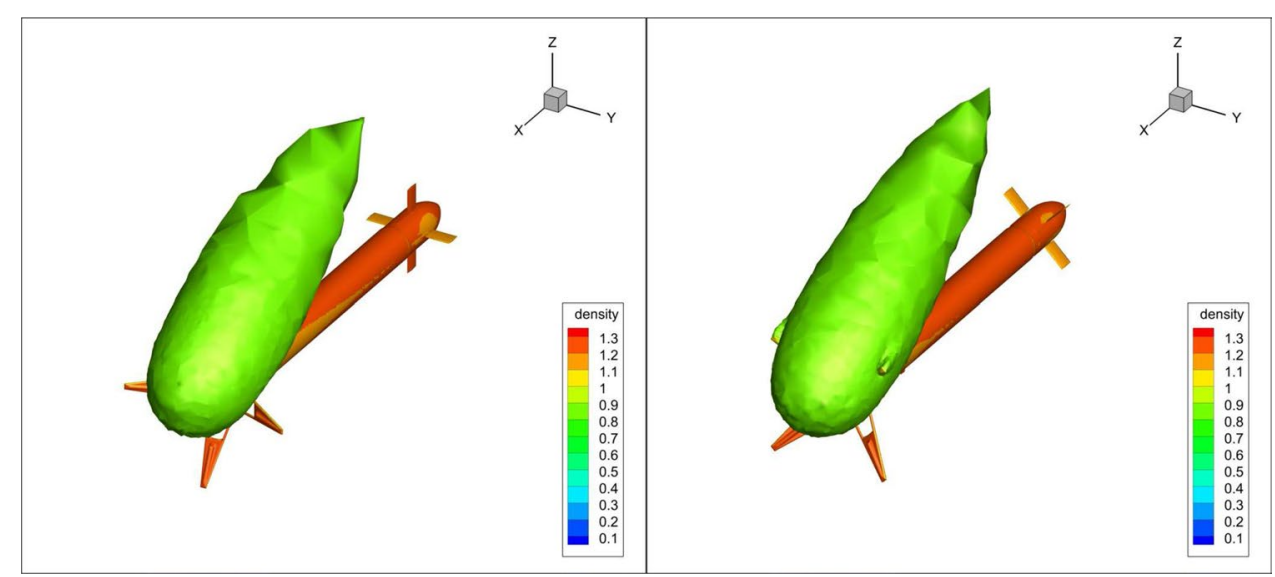


The deployment performance is, due to the AoR dependency of the aerodynamic flow conditions, dependent on the AoR. As mentioned in previous sections, the leeward oriented landing legs experience lower aerodynamic forces, than the windward oriented legs. In the context of this study, this load imbalance leads to a different total deployment time. The comparison of the deployment performance for an AoR of $0^{\circ}$ and an AoR of $45^{\circ}$ shows that the deployment with an AoR of $45^{\circ}$ takes longer, although two legs in comparison to one leg are partially covered by the engines plume. This effect can be traced back to the limited pneumatic power, which is not able to provide the same mass flow rate for all leg actuators in both flight orientations (Fig. 15).

\subsection{Influence of approach flight domain parameters on deployment performance}

The Approach Flight Domain parameters are the main contributor to the external loads, which the pneumatic system needs to overcome for a successful deployment. CALLISTO generates axial and lateral accelerations during it's approach phase, which partially supports the deployment. These accelerations are proportional to the hinge torque equation 20.

Whereas vehicle inertia forces directly act on the leg's center of gravity, the AoA and altitude vs. velocity profile $H$. vs. $V$ indirectly takes influence on the aerodynamic forces. While AoA is an input for the local aerodynamic coefficient $C_{\mathrm{M}}^{h, i}$, the $H$. vs. $V$. profile defines the aerodynamic pressure $q_{\infty}$, which both are inputs for the aerodynamic torque, as shown in Eq. 18 . In addition, AoA $\neq 180^{\circ}$ creates an asymmetric flow field around the vehicle, and, hence contributes to asynchronous deployment.

Besides, as described in Sect. 3.4, the AoR of CALLISTO defines the plume orientation, or rather, which landing leg is hit by the engine plume. The radial deflection of the plume,

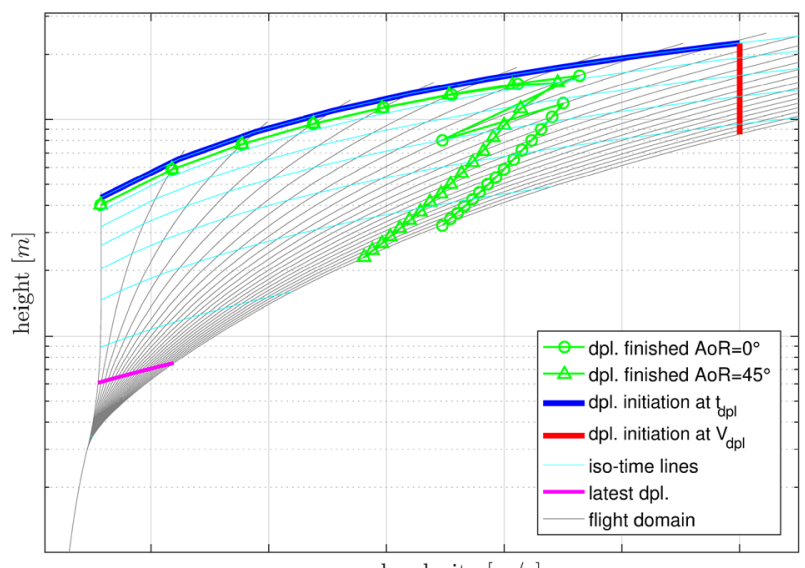

approach velocity $[\mathrm{m} / \mathrm{s}$

Fig. 15 Deployment performance difference between $A o R=0^{\circ}$ and $\mathrm{AoR}=45^{\circ}$ and hence the radial hit point on the landing leg, is defined by the AoA, and approach velocity. Consequently, these two parameters define in combination the heat flux that is introduced into the legs structure.

In the scope of this study, it is acceptable to consider each flight domain parameter individually. Nonetheless, coupled deployment and trajectory analysis are necessary, to achieve a holistic understanding of flight domain parameter changes.

To assess the reduction of deployment time, the AoA is set to $0^{\circ}$ for the whole flight duration. In this case, the flow field around the vehicle is symmetric and, therefore, the aerodynamic forces acting on the legs are symmetric and balanced. Since the deployment system provides same pneumatic power to all legs equally, the deployment conducts almost synchronous. As a result, the deployment time reduces, because all legs are exposed to the low-density plume, instead of one single leg for unsymmetrical flow conditions.

In contrast, if the vehicle's lateral accelerations is set to zero, longer deployment phases can be expected, because the lateral acceleration term in Eq. 20 is also set to zero. Both effects are reflected in the deployment performance in Fig. 16.

\subsection{Influence of thrust level on deployment performance}

During the approach phase, when the engine is on, the deflected engine plume envelopes the landing legs. Due to the decrease in pressure, the local aerodynamic coefficients $\underline{C}_{\mathrm{M}}^{h}$ of landing legs covered with plume are in this case lower, compared to landing legs that are submerged to the undisturbed flow [9].

To assess the engine plume deployment dynamics interaction, three possible thrust levels of $0 \%, 40 \%$, and $100 \%$,

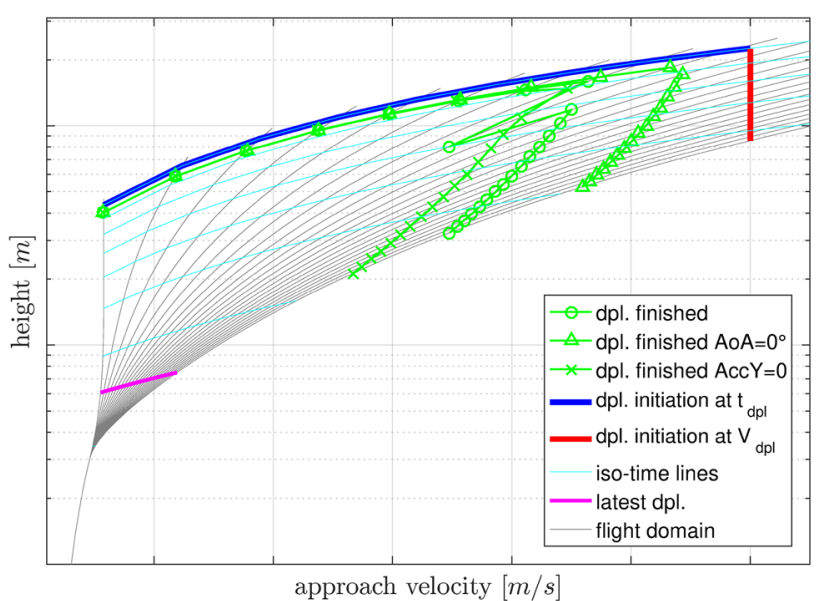

Fig. 16 Deployment performance for reduction of AoA $0^{\circ}$ 


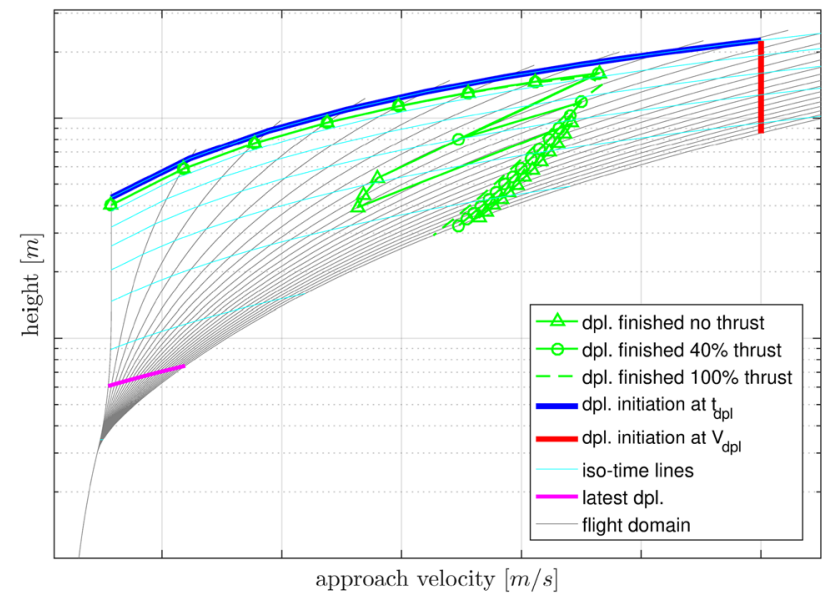

Fig. 17 Comparison of deployment performance with thrust levels of $0 \%, 40 \%$, and $100 \%$

have been investigated and applied on the approach flight domain. As a result, approach flights with low velocity show no difference in deployment performance, because the aerodynamic forces are negligible, compared with pneumatic forces. Whereas approach flights with higher velocities, lead to higher aerodynamic forces and, therefore, to shorter deployment durations in case the engine is ignited. In contrast, a deployment without beneficial reduction of aerodynamic coefficient, through engine plume flow properties, extends the deployment time. In this case, the limitation factor is the Push-Off mechanism torque, which cannot overcome initial aerodynamic torque, so that the deployment movement is initiated later. This effect is visualized in Fig. 17.

\section{Summary and conclusion}

On the example of CALLISTO VTVL vehicle the deployment dynamics are investigated. For this purpose, a landing leg deployment simulator, that mainly includes an extensive aerodynamic database, an approach flight domain database and a pneumatic deployment system model, is developed.

First, deployment dynamics of a single leg under consideration of aerodynamics and inertial forces are analyzed. It is shown, that the leg's pressure depends on pneumatic system parameters as well as on the external load, and, hence, internal and external loads try to attain an equilibrium during deployment. Furthermore, the analysis of the deployment of four legs show that due to the pneumatic circuit design legs deployment dynamics are mutually dependent. Another product design parameter that influences the initial movement of the deployment is the initial push-off mechanism force.
The effects of landing leg deployment on the vehicle are investigated. Specially, the disturbance forces and torques due to asymmetric flow conditions, which are mainly driven by AoA and AoR, are presented. These deployment induced disturbances need to be compensated by GNC-system. Beside the effects of design parameter on the deployment, this study examined the influence of the approach flight trajectory parameter on the deployment performance of CALLISTO. In this perimeter it is shown that the deployment duration mostly depends on AoA and, whether the engine plume surrounds the vehicle.

To sum up, the outcome of the performed analysis is that the deployment dynamics depend on trajectory parameters as well as on product parameters. Both contributes to the deployment dynamics in a non-linear manner, and needs to be adjusted, so that on the one hand the GNC system can handle the deployment induced loads and on the other hand the landing leg system can maintain its structural and functional integrity.

Funding Open Access funding enabled and organized by Projekt DEAL.

Open Access This article is licensed under a Creative Commons Attribution 4.0 International License, which permits use, sharing, adaptation, distribution and reproduction in any medium or format, as long as you give appropriate credit to the original author(s) and the source, provide a link to the Creative Commons licence, and indicate if changes were made. The images or other third party material in this article are included in the article's Creative Commons licence, unless indicated otherwise in a credit line to the material. If material is not included in the article's Creative Commons licence and your intended use is not permitted by statutory regulation or exceeds the permitted use, you will need to obtain permission directly from the copyright holder. To view a copy of this licence, visit http://creativecommons.org/licenses/by/4.0/.

\section{References}

1. Dumont, E., Ishimoto, S., Tatiossian, P., Klevanski, J., Reimann, B., Ecker, T., Witte, L., Riehmer, J., Sagliano, M., Vincenzino, S.G., Petkov, I., Rotärmel, W., Schwarz, R., Seelbinder, D., Markgraf, M., Sommer, J., Pfau, D., Martens, H.: CALLISTO: a demonstrator for reusable launcher key technologies. Trans. Jpn. Soc. Aeronaut. Space Sci. Aerosp. Technol. 19(1), 106-115 (2021). https://doi.org/10.2322/tastj.19.106

2. Vila, J., Hassin, J.: Technology acceleration process for the THEMIS low cost and reusable prototype. (2019). https://doi. org/10.13009/EUCASS2019-97

3. Dumont, E., Ecker, T., Chavagnac, C., Witte, L., Windelberg, J., Klevanski, J., Giagkozoglou, S.: CALLISTO-reusable VTVL launcher first stage demonstrator. In: Space Spropulsion Conference 2018 (2018). https://elib.dlr.de/119728/

4. Vincenzino, S.G., Rotärmel, W., Petkov, I., Elsäßer, H., Dumont, E., Witte, L., Schröder, S.: Reusable structures for callisto. In: 8th European Conference for Aeronautics and Space Sciences (EUCASS) (2019). https://elib.dlr.de/129444/

5. Bohl, W., Elmendorf, W.: Technische Strömungslehre Stoffeigenschaften Von Flüssigkeiten und Gasen, Hydrostatik, Aerostatik, Inkompressible Strömungen, Kompressible Strömungen, 
Strömungsmesstechnik, 15, überarbeitete und erweiterte, auflage Vogel Business Media, Wuürzburg (2013)

6. Beater, Peter: Pneumatic Drives: System Design. Modelling and Control. Springer, Berlin (2007)

7. Klevanski, J., Ecker, T., Riehmer, J., Reimann, B., Dumont, E., Chavagnac, C.: Aerodynamic studies in preparation for CALLISTO-reusable VTVL launcher first stage demonstrator. In: 69th International Astronautical Congress (IAC) (2018)
8. Langer, S., Schwöppe, A., Kroll, N.: The DLR flow solver TAUstatus and recent algorithmic developments. In: 52nd Aerospace Sciences Meeting (2014). https://elib.dlr.de/90979/

9. Ecker, T., Karl, S., Dumont, E., Stappert, S., Krause, D.: Numerical study on the thermal loads during a supersonic rocket retropropulsion maneuver. J. Spacecr. Rocket. 57(1), 131-146 (2020). https://doi.org/10.2514/1.A34486 